\title{
Dynamics of Entanglement Transfer Through Multipartite Dissipative Systems
}

\author{
C.E. López ${ }^{1,2}$, G. Romero ${ }^{1,3}$ and J.C. Retamal ${ }^{1,2}$ \\ ${ }^{1}$ Departamento de Física, Universidad de Santiago de Chile, USACH, Casilla 307 Correo 2 Santiago, Chile \\ ${ }^{2}$ Center for the Development of Nanoscience and Nanotechnology, 9170124, Estación Central, Santiago, Chile \\ ${ }^{3}$ Departamento de Química Física, Universidad del País Vasco - Euskal Herriko Unibertsitatea, Apdo. 644, 48080 Bilbao, Spain
}

(Dated: April 27, 2022)

\begin{abstract}
We study the dynamics of entanglement transfer in a system composed of two initially correlated three-level atoms, each located in a cavity interacting with its own reservoir. Instead of tracing out reservoir modes to describe the dynamics using the master equation approach, we consider explicitly the dynamics of the reservoirs. In this situation, we show that the entanglement is completely transferred from atoms to reservoirs. Although the cavities mediate this entanglement transfer, we show that under certain conditions, no entanglement is found in cavities throughout the dynamics. Considering the entanglement dynamics of interacting and non-interacting bipartite subsystems, we found time windows where the entanglement can only flow through interacting subsystems, depending on the system parameters.
\end{abstract}

PACS numbers: 03.65.Yz, 03.65.Ud, 03.67.Mn

\section{INTRODUCTION}

Entanglement has emerged as a central physical resource for Quantum Information Theory [1]. For processing quantum information, physical architectures are expected to be composed of multipartite quantum systems. Understanding how quantum entanglement is transferred between the parties has motivated several contributions in recent years [2 [6]. One issue is entanglement flow through individual parties and the whole multipartite system [2]. Another interesting problem is entanglement transfer between qubits and its relation to energy [3]. In addition, we have the study of the entanglement transfer between non-interacting qubits, leading to conservation rules for entanglement depending on how qubits are initially correlated [4]. Entanglement transfer from atoms to cavity modes leading to entanglement revivals has been studied in [5]. Entanglement transference from two cavities to their corresponding reservoirs allowed the description of Entanglement Sudden Death as opposed to Entanglement Sudden Birth [6].

An interesting problem in this context, is entanglement flow between parties in a multipartite system, including their dissipative mechanisms. In this work, we study the entanglement dynamics of two initially correlated atoms placed in two non-interacting leaky cavities, each connected to its own reservoir. To achieve this, we developed a hybrid analytical approach for finding the quantum dynamics of the atom-cavity-reservoir system. Unlike the master equation approach, our method allows us to include the reservoir dynamics, thus preventing information loss due to trace operations. We study the evolution of entanglement in different non-interacting bipartite subsystems, such as atom-atom, cavity-cavity and reservoir-reservoir. We show that the entanglement initially contained in the atomic subsystem is completely transferred into the reservoir-reservoir subsystem. Although cavities are the bridge connecting atoms to reservoirs, we show that they may not be entangled through- out the dynamics. Moreover, depending on the initial state, quantum dynamics may led to a situation where no entanglement exists in any of these three subsystems. In this case, we extend the study to other interacting and non-interacting subsystems.

This paper is organized as follows: in Section II we develop a hybrid analytical method to find the quantum dynamics. In Sec III, we study the dynamics of entanglement transfer. In Section I V we present our concluding remarks.

\section{ATOM-CAVITY-RESERVOIR DYNAMICS}

Our model considers two independent subsystems, each formed by a three-level atom inside a leaky QED cavity. Each atom interacts with a single mode of frequency $\omega$ of the quantized electromagnetic field and a classical field with frequency $\nu$ in a Raman configuration, as shown in Fig. 1. The quantum mode couples level $|g\rangle$ and $|c\rangle$ while classical field couples levels $|e\rangle$ and $|c\rangle$. Assuming no direct coupling between cavities, the dynamics of each atom-cavity-reservoir system can be studied individually. Neglecting effects of spontaneous emission from leves $|c\rangle$ and $|e\rangle$, the Hamiltonian describing this system can be conveniently written, in the rotating wave approximation, as:

$$
\begin{aligned}
\hat{H}= & \hbar \Delta|c\rangle\langle c|-\hbar \delta| e\rangle\langle e|-\hbar \sum_{k=1}^{N}\left(\omega-\omega_{k}\right) \hat{b}_{k}^{\dagger} \hat{b}_{k} \\
& +\hbar \Omega(|c\rangle\langle e|+| e\rangle\langle c|)+\hbar g\left(\hat{a}|c\rangle\left\langle g\left|+\hat{a}^{\dagger}\right| g\right\rangle\langle c|\right) \\
& +\hbar \sum_{k=1}^{N}\left(g_{k} \hat{a} \hat{b}_{k}^{\dagger}+g_{k}^{*} \hat{b}_{k} \hat{a}^{\dagger}\right) .
\end{aligned}
$$

Here, $\hat{a}\left(\hat{a}^{\dagger}\right)$ annihilates (creates) a photon with frequency $\omega$ in the cavity mode, operator $\hat{b}_{k}\left(\hat{b}_{k}^{\dagger}\right)$ annihilates 


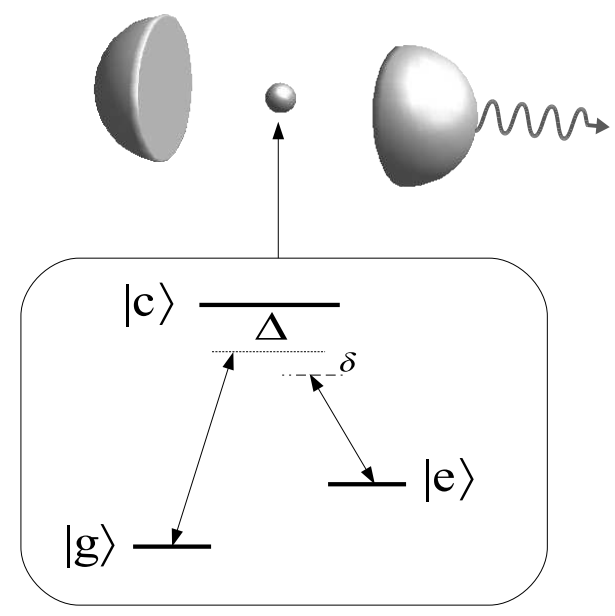

FIG. 1. Scheme of a three-level atom inside a cavity coupled to a reservoir.

(creates) a photon with frequency $\omega_{k}$ in $k$-th mode of the reservoir. We have defined $\Delta=\omega_{c g}-\omega$, with $\omega_{c g}$ is the frequency difference between levels $|c\rangle$ and $|g\rangle$, detuning $\delta=\omega_{c e}-\nu-\Delta$. The coupling strength between the classical field and the atom is $\Omega$ and we have explicitly written the system-bath interaction with a linear coupling with coupling constant $g_{k}$. In the usual master equation approach, considering the Markov approximation and an infinite number of bath oscillators, we can describe the dynamics of the atom-cavity system by

$$
\dot{\hat{\rho}}=-i\left[\hat{H}^{\prime}, \hat{\rho}\right]+\frac{\kappa}{2}\left(2 \hat{a}^{\dagger} \hat{\rho} \hat{a}-\hat{a}^{\dagger} \hat{a} \hat{\rho}-\hat{\rho} \hat{a}^{\dagger} \hat{a}\right),
$$

where $\hat{H}^{\prime}$ corresponds to Hamiltonian (1) without terms involving the reservoir.

In such an approach, only atom-cavity dynamics is described, while the reservoirs are traced out. This results in loss of quantum correlations between reservoirs and other parties.

In what follows, we will develop a method to find the dynamics, including explicitly the reservoir's degrees of freedom. In this manner we preserve all the information about quantum correlations in the system

To address this problem, we develop a hybrid analytical approach to find the dynamics. First, we will follow the method described for obtaining the quantum dynamics and the entanglement properties of inhomogeneouslycoupled systems [7, 8]. This method consists of inspecting the Hilbert space occupied for the quantum system throughout the evolution and implementing a truncation criteria based on a probabilistic argument. Then, to find explicitly analytical expressions for the quantum dynamics, we follow the well-known quantum trajectory method [10].

Let us consider the case of a single initial excitation contained in the atomic subsystem. That is,

$$
\left|\psi_{0}\right\rangle=|e\rangle_{a} \otimes|0\rangle_{c} \otimes|\overline{\mathbf{0}}\rangle_{r} \equiv|e 0 \overline{\mathbf{0}}\rangle
$$

where, $|0\rangle_{c}$ corresponds to the vacuum of the cavity and $|\overline{\mathbf{0}}\rangle_{r} \equiv \prod_{k}\left|0_{k}\right\rangle$ denotes the vacuum of the reservoir. Now we have to look for the accessible Hilbert space for the compound system when starting from this initial condition. We can find this by following the action of Hamiltonian $\hat{H}_{I I}$ on the initial state $\left|\psi_{0}\right\rangle$. It is not difficult to realize that a portion of the Hilbert space connected by Hamiltonian (10) is given by states $\{|e 0 \overline{\mathbf{0}}\rangle,|c 0 \overline{\mathbf{0}}\rangle,|g 1 \overline{\mathbf{0}}\rangle\}$. The one photon excitation in state $|g 1 \overline{\mathbf{0}}\rangle$ is transferred to bath modes through Hamiltonian (11) as follows:

$$
\hat{H}_{I I}|g 1 \overline{\mathbf{0}}\rangle=\hbar g|c 0 \overline{\mathbf{0}}\rangle+\hbar N_{0}\left|g 0 \overline{\mathbf{1}}_{0}\right\rangle
$$

where we have defined the state:

$$
\left|\overline{\mathbf{1}}_{0}\right\rangle_{r} \equiv \frac{1}{N_{0}} \sum_{k=1}^{n} g_{k}\left|1_{k}\right\rangle_{r}
$$

with $N_{0}=\sqrt{\sum_{k=1}^{n}\left|g_{k}\right|^{2}}$, and $\left|1_{k}\right\rangle_{r}$ corresponds to the state having one photon in the $k$-th reservoir mode and zero photon in the remaining modes. The state in Eq. (5) is a collective state of the reservoir having a single excitation. The $\left|g 0 \overline{1}_{0}\right\rangle$ collective state evolves under the interaction part in Hamiltonian (1), back to states $|g 1 \overline{\mathbf{0}}\rangle$ and $|c 0 \overline{\mathbf{0}}\rangle$. However, this is no longer true when considering the action of the reservoir Hamiltonian on reservoir states. Taking the vacuum state of the reservoir, we have

$$
\sum_{k}^{N}\left(\omega-\omega_{k}\right) \hat{b}_{k}^{\dagger} \hat{b}_{k}|\overline{\mathbf{0}}\rangle_{r}=0 .
$$

For $\left|\overline{1}_{0}\right\rangle_{r}$, free energy term leads to

$$
\left|\Phi_{1}\right\rangle=\frac{1}{N_{0}} \sum_{k}^{N} g_{k}\left(\omega-\omega_{k}\right)\left|1_{k}\right\rangle_{r} .
$$

Although this state is different from $\left|\overline{1}_{0}\right\rangle_{r}$ in Eq.(15), it can be written as a superposition of this state and a state $\left|\overline{1}_{1}\right\rangle_{r}$ orthogonal to $\left|\overline{1}_{0}\right\rangle_{r}$. That is,

$$
\left|\overline{\mathbf{1}}_{1}\right\rangle_{r}=\frac{1}{N_{1}}\left[\left|\Phi_{1}\right\rangle-\left\langle\overline{\mathbf{1}}_{0} \mid \Phi_{1}\right\rangle\left|\overline{\mathbf{1}}_{0}\right\rangle_{r}\right],
$$

where $N_{1}=\left(\left\langle\Phi_{1} \mid \Phi_{1}\right\rangle-\left|\left\langle\overline{\mathbf{1}}_{0} \mid \Phi_{1}\right\rangle\right|^{2}\right)^{1 / 2}$.

The new generated state $\left|\overline{\mathbf{1}}_{1}\right\rangle_{r}$ leads to other orthogonal states having one excitation through the action of the reservoir terms of the Hamiltonian [7]. In consequence, the accessible Hilbert space for the overall system initially prepared in state (3), can be written in a collective basis spanned by the set of orthogonal states

$$
\left\{|e 0 \overline{\mathbf{0}}\rangle,|c 0 \overline{\mathbf{0}}\rangle,|g 1 \overline{\mathbf{0}}\rangle,\left|g 0 \overline{\mathbf{1}}_{0}\right\rangle,\left|g 0 \overline{\mathbf{1}}_{1}\right\rangle,\left|g 0 \overline{\mathbf{1}}_{2}\right\rangle, \ldots\right\} .
$$

Therefore, the atom-cavity-reservoir system governed by Hamiltonian (1) evolves to

$$
\begin{aligned}
\left|\psi_{t}\right\rangle= & E_{t}|e 0 \overline{\mathbf{0}}\rangle+C_{t}|c 0 \overline{\mathbf{0}}\rangle+G_{t}|g 1 \overline{\mathbf{0}}\rangle \\
& +R_{0, t}\left|g 0 \overline{\mathbf{1}}_{0}\right\rangle+R_{1, t}\left|g 0 \overline{\mathbf{1}}_{1}\right\rangle+\ldots,
\end{aligned}
$$


where $R_{j, t}$ is the time-dependent probability amplitude of the state $\left|g 0 \overline{\mathbf{1}}_{j}\right\rangle$. This state can conveniently written defining one-excitation collective state for the reservoir

$$
|\overline{\mathbf{1}}\rangle=\frac{1}{R_{t}}\left(R_{0, t}\left|\overline{\mathbf{1}}_{0}\right\rangle+R_{1, t}\left|\overline{\mathbf{1}}_{1}\right\rangle+\ldots\right),
$$

where,

$$
R_{t}=\sqrt{\left|R_{0, t}\right|^{2}+\left|R_{1, t}\right|^{2}+\ldots}
$$

corresponds to the probability amplitude of having one excitation in the reservoir modes.

In this manner, the dynamics of the atom-cavityreservoir system can be described in terms of a threequbit system in the basis: $\{|e\rangle,|g\rangle\} \otimes\{|0\rangle,|1\rangle\} \otimes\{|\overline{\mathbf{0}}\rangle,|\overline{\mathbf{1}}\rangle\}$. Notice that we have assumed the high detuning regime $(\Delta \gg \Omega, g)$, so the electronic level $|c\rangle_{a}$ is only virtually populated and the evolution described in Eq. (10) can now be written as

$$
\left|\psi_{t}\right\rangle=E_{t}|e 0 \overline{\mathbf{0}}\rangle+G_{t}|g 1 \overline{\mathbf{0}}\rangle+R_{t}|g 0 \overline{\mathbf{1}}\rangle .
$$

Probability amplitudes can be obtained by numerical diagonalization of Hamiltonian (11) where it is found that $C_{t} \sim 0$. It is worth to notice that this form of finding the quantum dynamics has close relation with the WeisskopfWigner procedure (see for example ref. [9]) where the reservoir is considered throughout the dynamics.

We are interested in finding analytical expressions for the temporal dependent coefficients in the wave function in Eq. (13). Unfortunately, this is not possible by solving the Schrödinger equation. However, if we know the reduced dynamics for the atom-cavity system, we can get information about the probability amplitude $R_{t}$. The reduced atom-cavity dynamics could be obtained by solving the corresponding master equation, or alternatively by using the quantum trajectory method (see for example [10] and references within). By using this approach, we obtain the reduced density matrix for the atom-cavity system as:

$$
\begin{aligned}
\hat{\rho}(t)= & \left|E_{t}\right|^{2}|e 0\rangle\left\langle\left. e 0|+| G_{t}\right|^{2} \mid g 1\right\rangle\langle g 1| \\
& +E_{t} G_{t}^{*}|e 0\rangle\left\langle g 1\left|+E_{t}^{*} G_{t}\right| g 1\right\rangle\langle e 0| \\
& +\left|R_{t}\right|^{2}|g 0\rangle\langle g 0|
\end{aligned}
$$

The probability amplitudes obtained through the quantum trajectory approach are

$$
\begin{aligned}
E_{t} & =\left\{\cos (\bar{\Omega} t)+\frac{\kappa}{4 \bar{\Omega}} \sin (\bar{\Omega} t)\right\} e^{-\frac{1}{4} \kappa t}, \\
G_{t} & =\frac{i g_{\text {eff }}}{\bar{\Omega}} \sin (\bar{\Omega} t) e^{-\frac{1}{4} \kappa t},
\end{aligned}
$$

where $4 \bar{\Omega}^{2}=4 g_{\text {eff }}^{2}-\kappa^{2} / 4$ and $g_{\text {eff }}=g \Omega / \Delta$. For simplicity, here we have set $\delta=\left(g^{2}-\Omega^{2}\right) / \Delta$. From these expressions we obtain the probability amplitude $R_{t}$ as:

$$
\left|R_{t}\right|=\sqrt{1-\left|E_{t}\right|^{2}-\left|G_{t}\right|^{2}} .
$$

On the other hand, since $R_{t}$ is by definition a realpositive number $\left(\left|R_{t}\right|=R_{t}\right)$, we can say that the dynamics is given by Eq.(13) with $E_{t}, G_{t}$ and $R_{t}$, defined in Eqs. (15) and (16).

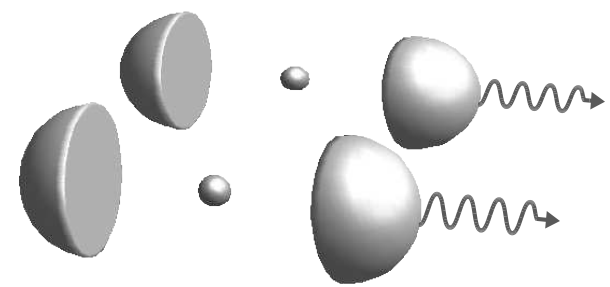

FIG. 2. Scheme of two initially entangled atoms, each located in a leaky cavity.

\section{DYNAMICS OF ENTANGLEMENT TRANSFER}

Having described the dynamics of the atom-cavityreservoir subsystem, we now focus on the problem of two initially entangled atoms, each located in a leaky cavity, as shown in Fig. 2. This entangled state between distant atoms have received much attention in the last years due to its possible applications in Quantum Communication [11 17]. In this sense, the present study might be relevant. On the other hand, this coherent manipulation of atoms and cavities could also be extended into a trapped ions system. For example in the experimental setup described in reference [18], a single trapped ion is coupled to a high-finesse cavity. Further improvements in this experiment could consider a second cavity coupled to a second ion. In this case, the same dynamics we are describing in this section can be found in this system. Entanglement between the electronic levels of the ions can be prepared using the center-of-mass mode.

In our system, since there is no interaction between both atom-cavity-reservoir subsystems, the only link is provided by the entanglement between the atoms. Within this scenario, we are interested in the study of how the entanglement, initially shared by the two atoms, is transferred to other parties. To do this, we first consider an initial entangled atomic state given by

$$
\left|\Psi_{0}\right\rangle=\left(\alpha|g g\rangle_{12}+\beta|e e\rangle_{12}\right)|00\rangle_{12}|\overline{\mathbf{0}} \overline{\mathbf{0}}\rangle_{12},
$$

where cavities and reservoirs are in the vacuum state. Following the previous single atom-cavity-reservoir analysis, the initial state $\left|\Psi_{0}\right\rangle$ evolves to:

$$
\left|\Psi_{t}\right\rangle=\alpha|g 0 \overline{0}\rangle_{1} \otimes|g 0 \overline{0}\rangle_{2}+\beta\left|\psi_{t}\right\rangle_{1} \otimes\left|\psi_{t}\right\rangle_{2},
$$

where $\left|\psi_{t}\right\rangle$ is given in Eq. (13). By tracing out the degrees of freedom of cavities and reservoirs we are led to the atom-atom dynamics:

$$
\begin{aligned}
\rho_{a_{1} a_{2}}= & \beta^{2}\left|E_{t}\right|^{4}|e e\rangle\left\langle\left. e e|+\alpha \beta| E_{t}\right|^{2}(|e e\rangle\langle g g|+| g g\rangle\langle e e|)\right. \\
& +\beta^{2}\left|E_{t}\right|^{2}\left(\left|G_{t}\right|^{2}+\left|R_{t}\right|^{2}\right)(|e g\rangle\langle e g|+| g e\rangle\langle g e|) \\
& +\left(\alpha^{2}+\beta^{2}\left(\left|G_{t}\right|^{2}+\left|R_{t}\right|^{2}\right)^{2}\right)|g g\rangle\langle g g| .
\end{aligned}
$$

Atomic entanglement can be evaluated by using the concurrence [19], leading to:

$$
\mathcal{C}_{a_{1} a_{2}}(t)=\max \left\{0,-2 \lambda_{-}^{a_{1} a_{2}}\right\},
$$


where the negative eigenvalue of the partial transpose matrix [20, 21] of $\rho_{a_{1} a_{2}}$ is given by:

$$
\lambda_{-}^{a_{1} a_{2}}=\beta\left|E_{t}\right|^{2}\left(\beta\left(1-\left|E_{t}\right|^{2}\right)-\alpha\right) .
$$

The entanglement flow from atoms to other parties is obtained from cavity-cavity and reservoir-reservoir reduced systems, respectively. The reduced cavity-cavity system is described by:

$$
\begin{aligned}
\rho_{c_{1} c_{2}}= & \beta^{2}\left|G_{t}\right|^{4}|11\rangle\left\langle\left. 11|+\alpha \beta| G_{t}\right|^{2}(|00\rangle\langle 11|+| 11\rangle\langle 00|)\right. \\
& +\beta^{2}\left|G_{t}\right|^{2}\left(\left|E_{t}\right|^{2}+\left|R_{t}\right|^{2}\right)(|10\rangle\langle 10|+| 01\rangle\langle 01|) \\
& +\left(\alpha^{2}+\beta^{2}\left(\left|E_{t}\right|^{2}+\left|R_{t}\right|^{2}\right)^{2}\right)|00\rangle\langle 00|,
\end{aligned}
$$

while for the reduced reservoir-reservoir system we have:

$$
\begin{aligned}
\rho_{r_{1} r_{2}}= & \beta^{2}\left|R_{t}\right|^{4}|\overline{\mathbf{1}} \overline{\mathbf{1}}\rangle\left\langle\left.\overline{\mathbf{1}} \overline{\mathbf{1}}|+\alpha \beta| R_{t}\right|^{2}(|\overline{\mathbf{0}} \overline{\mathbf{0}}\rangle\langle\overline{\mathbf{1}} \overline{\mathbf{1}}|+| \overline{\mathbf{1}} \overline{\mathbf{1}}\rangle\langle\overline{\mathbf{0}} \overline{\mathbf{0}}|)\right. \\
& +\beta^{2}\left|R_{t}\right|^{2}\left(\left|E_{t}\right|^{2}+\left|G_{t}\right|^{2}\right)(|\overline{\mathbf{1}} \overline{\mathbf{0}}\rangle\langle\overline{\mathbf{1}} \overline{\mathbf{0}}|+| \overline{\mathbf{0}} \overline{\mathbf{1}}\rangle\langle\overline{\mathbf{0}} \overline{\mathbf{1}}|) \\
& +\left(\alpha^{2}+\beta^{2}\left(\left|E_{t}\right|^{2}+\left|G_{t}\right|^{2}\right)^{2}\right)|\overline{\mathbf{0}} \overline{\mathbf{0}}\rangle\langle\overline{\mathbf{0}} \overline{\mathbf{0}}| .
\end{aligned}
$$

The corresponding entanglement in these subsystems can also be calculated using the concurrence. The concurrences for cavities and reservoirs, respectively, are given by:

$$
\begin{aligned}
& \mathcal{C}_{c_{1} c_{2}}(t)=\max \left\{0,-2 \lambda_{-}^{c_{1} c_{2}}\right\}, \\
& \mathcal{C}_{r_{1} r_{2}}(t)=\max \left\{0,-2 \lambda_{-}^{r_{1} r_{2}}\right\}
\end{aligned}
$$

where the negatives eigenvalues of partial transposed matrices are given by:

$$
\begin{aligned}
& \lambda_{-}^{c_{1} c_{2}}=\beta\left|G_{t}\right|^{2}\left(\beta\left(1-\left|G_{t}\right|^{2}\right)-\alpha\right) \\
& \lambda_{-}^{r_{1} r_{2}}=\beta\left|R_{t}\right|^{2}\left(\beta\left(1-\left|R_{t}\right|^{2}\right)-\alpha\right) .
\end{aligned}
$$

We are now in a position to investigate the evolution of entanglement for different subsystems. Disentanglement dynamics depends on probability amplitudes $\alpha$ and $\beta$, as well as the decay constant $\kappa$ compared to the effective coupling $g_{\text {eff }}$. For example, an important case is an initial maximally entangled state. Fig. 3 shows the entanglement evolution for atoms, cavities and reservoir subsystems, starting from this state with $\alpha=\beta$ for $g_{\text {eff }}=5 \kappa$. For the chosen parameters, we observe that the entanglement exhibits asymptotic decay, with oscillations of atomic entanglement and cavities entanglement to finally be completely transferred into the reservoirs. These oscillations are expected because for $g_{\text {eff }}>\kappa$, there are many energy exchange between atomic and cavity subsystems before the energy is completely transferred to reservoirs. In addition, the entanglement between reservoirs appears at the same time as entanglement between atoms begins to decrease. This regime is reminiscent from what happens for an initial maximally entangled state under the action of a dissipative environment.

For cavities with no atoms and the initial state given by $|\Psi\rangle=\left(c_{0}|00\rangle+c_{1}|11\rangle\right)|\overline{\mathbf{0}} \overline{\mathbf{0}}\rangle$, it is well known that entanglement disappears at a finite time if $c_{1}>c_{0}$ [22]. Otherwise, if $c_{0}>c_{1}$, the entanglement decays asymptotically. Moreover, in ref. [6], finite-time disentanglement, known

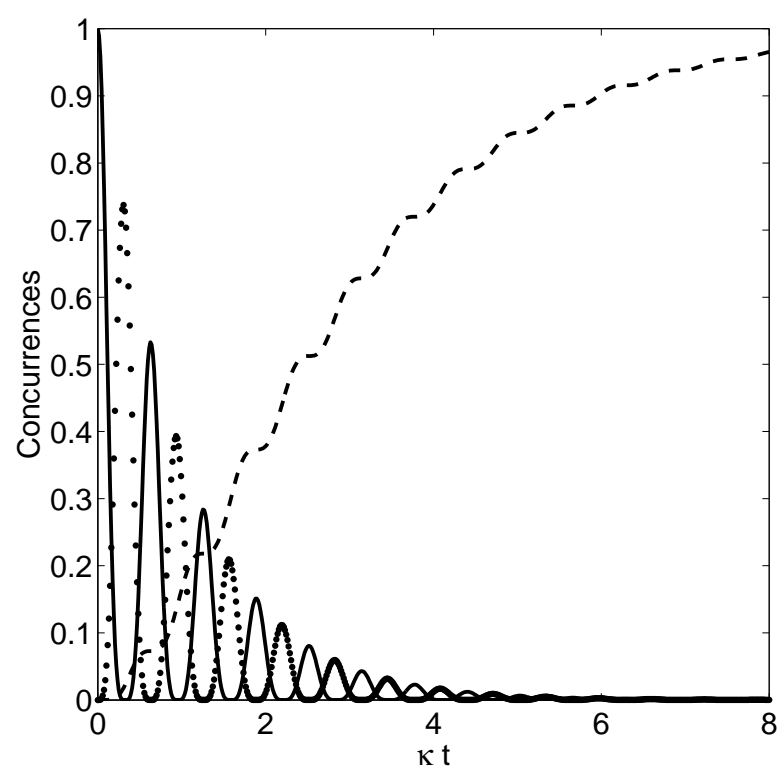

FIG. 3. Evolution of concurrence of subsystems: $a_{1} \otimes a_{2}$ (solid line); $c_{1} \otimes c_{2}$ (dashed line) and $r_{1} \otimes r_{2}$ (dot-dashed line) for an initial state of the form of Eq. (17) with $\alpha=\beta=1 / \sqrt{2}$ and $g_{\mathrm{eff}}=5 \kappa$.

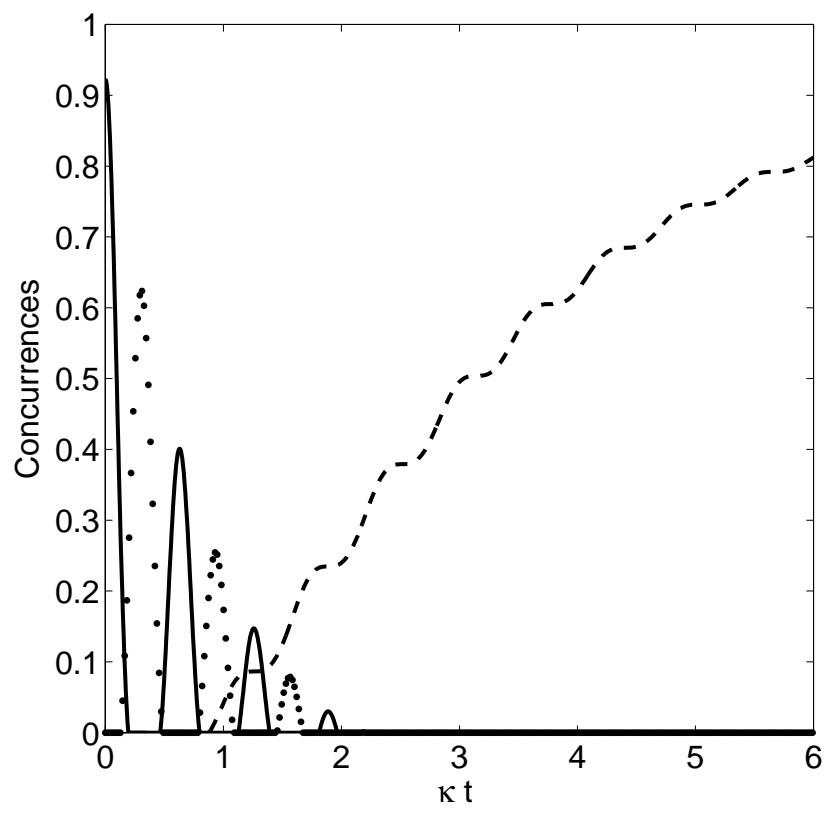

FIG. 4. Evolution of concurrence of subsystems: $a_{1} \otimes a_{2}$ (solid line); $c_{1} \otimes c_{2}$ (dots) and $r_{1} \otimes r_{2}$ (dashed line) for an initial state of the form of Eq. (17) with $\beta=1.5 \alpha$ and $g_{\text {eff }}=5 \kappa$.

as, entanglement sudden death (ESD) [23 25] is necessarily linked to a sudden birth of entanglement between reservoirs, called entanglement sudden birth (ESB). This behavior can also occur in our system by considering unbalanced states with $\beta>\alpha$. In Fig. 4, the evolution of the entanglement contained in the three subsystems 
is shown for $\beta>\alpha$ and $g_{\text {eff }}=5 \kappa$. We realize that es tanglement still experiences oscillations between cavitis and atoms, however, entanglement experiences sever: sudden deaths and entanglement sudden revivals (ESR whereas the reservoirs experience a sudden birth of eI tanglement.

These behaviors can in principle be quantitatively ur derstood from Eqs. (21), (26) and (27). For arbitrar effective coupling constant $g_{\text {eff }}$ and amplitudes $\alpha$ and $\beta$, is not possible to calculate the times at which ESD, ES or ESB appears. But for the case of reservoir entangl ment Eq. (27) says that for $\alpha>\beta$ there will always k an entanglement birth at time $t=0$. However, for $\beta>$ the reservoirs will remain unentangled unless:

$$
\left|R_{t}\right|^{2}>1-\frac{\alpha}{\beta} \text {. }
$$

We observe this is the behavior in Fig 4 where ES appears for a $t_{\mathrm{ESB}}>0$.

Some analytical calculations can be carried out in sp' cial regimes of $g_{\text {eff }}$ as compared to $\kappa$. In the strong coupling regime, when $g_{\text {eff }} \gg \kappa$ we have $\bar{\Omega} \approx g_{\text {eff }}$, so that:

$$
\begin{aligned}
& \left|E_{t}\right|^{2} \approx \cos ^{2}\left(g_{\text {eff }} t\right) e^{-\frac{1}{2} \kappa t} \\
& \left|G_{t}\right|^{2} \approx \sin ^{2}\left(g_{\text {eff }} t\right) e^{-\frac{1}{2} \kappa t} \\
& \left|R_{t}\right|^{2} \approx 1-e^{-\frac{1}{2} \kappa t}
\end{aligned}
$$

From Eqs. (21) and (26) we can explain the entanglement death and revivals zones for atoms and cavities in Fig. 4. Conditions for disentanglement in both subsystems are given by:

$$
\begin{aligned}
& 1-\cos ^{2}\left(g_{\mathrm{eff}} t\right) e^{-\frac{1}{2} \kappa t} \geq \frac{\alpha}{\beta} \\
& 1-\sin ^{2}\left(g_{\mathrm{eff}} t\right) e^{-\frac{1}{2} \kappa t} \geq \frac{\alpha}{\beta}
\end{aligned}
$$

In the strong coupling regime, we find time windows with no entanglement between atoms, that is, time windows between ESD and ESR times. These time windows for atoms happen at time intervals that can be different from those for cavities, or can overlap, depending on the ratio $\alpha / \beta$. At the same time the entanglement in both subsystems extinguish definitely because of dissipation given by the attenuation factor in the previous inequalities. This can be observed in Fig 5 , where evolutions of Eqs. (32) and (33) are shown compared to $\alpha / \beta$. Here we see that the attenuation factor $\exp (-\kappa t / 2)$ makes it possible to satisfy conditions (32) and (33), leading to complete disentanglement of atoms and cavities. In addition, from Eq. (28) we can evaluate the time for ESB in the reservoir subsystem, which is given by:

$$
t_{\mathrm{ESB}} \approx 2 \frac{1}{\kappa} \ln \left[\frac{\beta}{\alpha}\right]
$$

This time is twice the found for two entangled dissipative cavities studied in [6]. In the present case ESB also happens if $\beta>\alpha$.

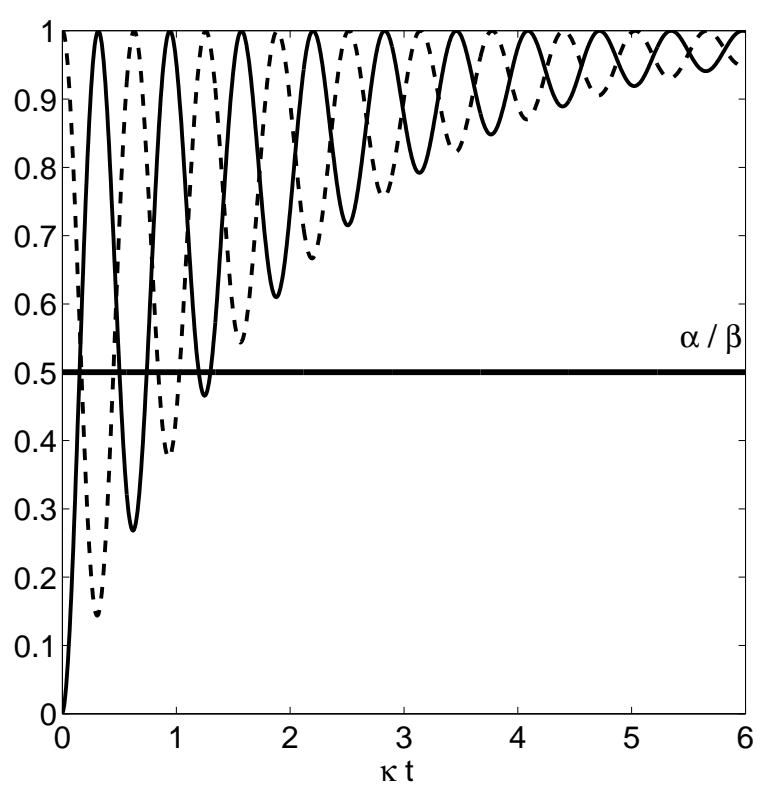

FIG. 5. Evolution of Eqs. (32) (solid line) and (33) (dashed line) for $g_{\text {eff }}=5 \kappa$. Dot-dashed line shows the value of $\alpha / \beta$.

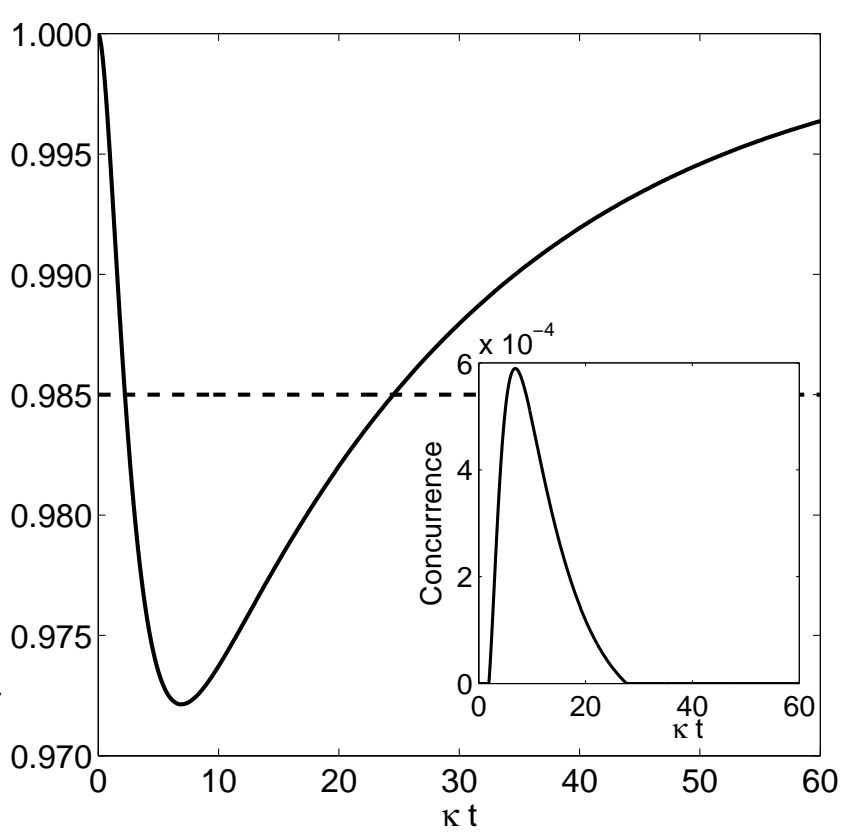

FIG. 6. Evolution of the left-hand side of Eq. (38) (solid line) for $g_{\mathrm{eff}}=0.1 \kappa$. Dashed line shows $\alpha / \beta=0.985$. The inset shows the concurrence $\mathcal{C}_{c_{1} c_{2}}(t)$ for the cavity-cavity subsystem.

On the other hand, in the weak coupling regime $g_{\text {eff }} \ll$ 
$\kappa$, we find that:

$$
\begin{aligned}
& \left|E_{t}\right|^{2} \approx\left(1+4 \gamma^{2}\right) e^{-4 \gamma^{2} \kappa t}-4 \gamma^{2} e^{-\kappa t+4 \gamma^{2} \kappa t} \\
& \left|G_{t}\right|^{2} \approx 4 \gamma^{2}\left(e^{-4 \gamma^{2} \kappa t}+e^{-\kappa t+4 \gamma^{2} \kappa t}-2 e^{-\kappa t / 2}\right) \\
& \left|R_{t}\right|^{2} \approx 1-\left(1+8 \gamma^{2}\right) e^{-4 \gamma^{2} \kappa t}+8 \gamma^{2} e^{-\kappa t / 2}
\end{aligned}
$$

where we have considered only up to second order in $\gamma=g_{\text {eff }} / \kappa$. From these equations we observe that unlike the strong coupling regime, in the weak coupling regime the entanglement dynamics is not oscillatory, that is, no entanglement revivals can be found in atoms or cavities. We expect that disentanglement between atoms be followed by entanglement birth in reservoirs. However entanglement between cavities depends on $\gamma$ and the ratio $\alpha / \beta$. This can be understood by considering Eqs. (26) and (36), such that the condition for disentanglement in the cavities is given by:

$$
1-4 \gamma^{2}\left(e^{-4 \gamma^{2} \kappa t}+e^{-\kappa t+4 \gamma^{2} \kappa t}-2 e^{-\kappa t / 2}\right) \geq \frac{\alpha}{\beta}
$$

The evolution of the left-hand side of the inequality is shown in Fig. 6. According to (38), this figure shows that cavities are entangled depending on the ratio $\alpha / \beta$. More precisely, cavities get entangled only while the curve is below the value of $\alpha / \beta$. In particular, for the case of $\alpha / \beta=0.985$ (dashed line) cavities get entangled only when the curve is below the dashed line, as shown in the inset where the cavity-cavity concurrence is plotted. When the ratio $\alpha / \beta$ is set to a value below the minimum of the left hand side in (38) cavities never become entangled. The particular case of Fig 6 happens for $\alpha / \beta<0.972$. In such a case the entanglement initially contained in the atomic subsystem is transferred directly to the reservoirs without entangling the cavities. This feature of entanglement dynamics can even be found outside the weak coupling regime as a function of $\gamma$ and $\alpha / \beta$. We can distinguish an entangled phase and an unentangled phase throughout the dynamics, which can be obtained from Eqs. (24) and (26). Fig. 7 shows the two phases for the cavities entanglement dynamics as a function of $\gamma$ and $\alpha / \beta$.

In the case of atom and reservoir subsystems, the non oscillatory behavior is shown in Fig 8 for $\gamma=0.1$ and $\alpha / \beta=2 / 3$. In such a case, the atom subsystem exhibits ESD, while the reservoir subsystem exhibits ESB. The time at which ESD and ESB happen depends on the ratio $\alpha / \beta$ for a fixed $\gamma$. In the weak coupling regime, and for values of $\gamma$ that allows to neglect corrections in $\gamma^{2}$ in both Eqs. (35) and (37), we have:

$$
\begin{aligned}
& t_{\mathrm{ESD}} \approx\left(\frac{1}{4 \gamma^{2}}\right) \frac{1}{\kappa} \ln \left[\frac{\beta}{\beta-\alpha}\right], \\
& t_{\mathrm{ESB}} \approx\left(\frac{1}{4 \gamma^{2}}\right) \frac{1}{\kappa} \ln \left[\frac{\beta}{\alpha}\right] .
\end{aligned}
$$

From these Eqs. we observe that the ESB time can occur before, simultaneously or after the ESD. For example, in Fig. 9. entanglement dynamics is shown for $\gamma=0.1$ and

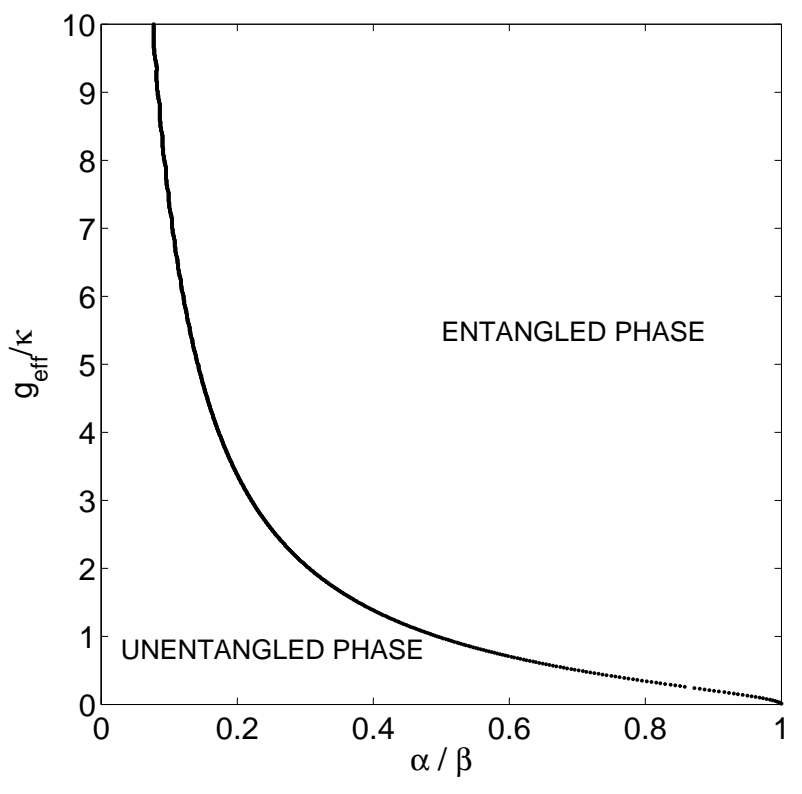

FIG. 7. Diagram showing the entangled/unentangled phases of the cavity-cavity subsystem as a function of coefficients $g_{\text {eff }} / \kappa$ and $\alpha / \beta$.

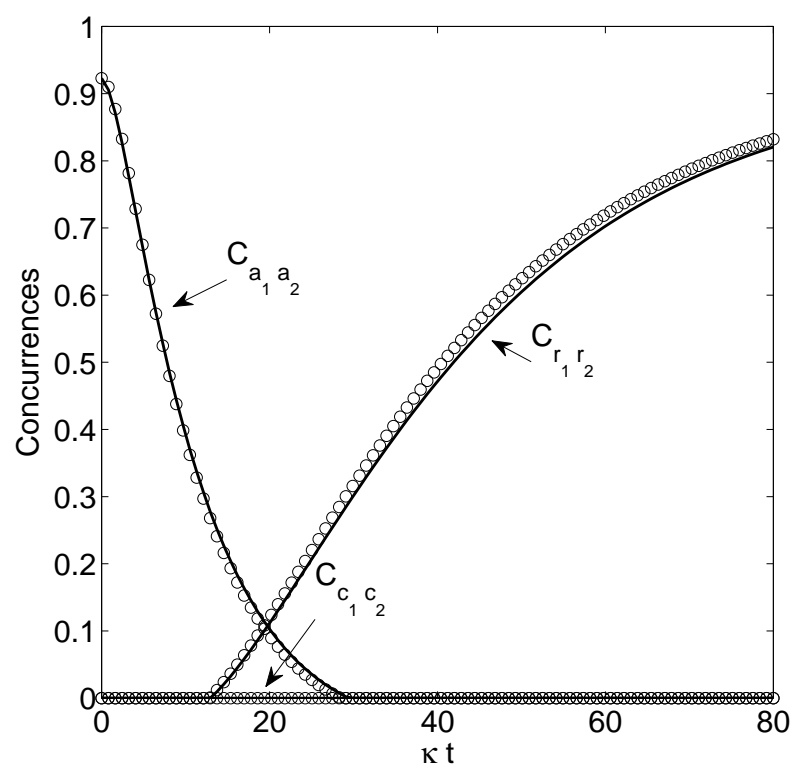

FIG. 8. Evolution of concurrence of subsystems $a_{1} \otimes a_{2}, c_{1} \otimes$ $c_{2}$ and $r_{1} \otimes r_{2}$, using Eqs. (35)-(37) (solid lines) and exact calculations (circles). Parameter are $\alpha / \beta=2 / 3$ and $g_{\text {eff }}=$ $0.1 \kappa$.

$\beta=3 \alpha$. In such a figure we observe that there is a time window for which no entanglement is found in the three subsystems. Using Eqs.(39) and (40), we can calculate the size of this time window, leading to:

$$
\Delta t_{W} \approx\left(\frac{1}{4 \gamma^{2}}\right) \frac{1}{\kappa} \ln \left[\frac{\beta}{\alpha}-1\right]
$$




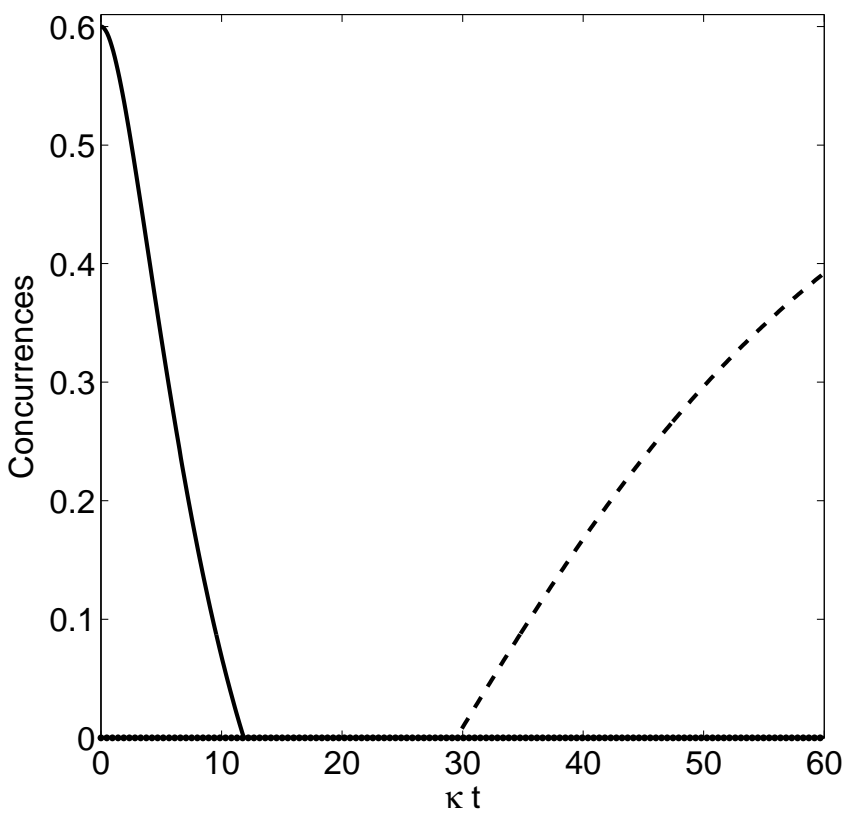

FIG. 9. Evolution of concurrence of the subsystems $a_{1} \otimes a_{2}$ (solid line), $c_{1} \otimes c_{2}$ (circles), $r_{1} \otimes r_{2}$ (dashed line) for $\beta=3 \alpha$ and $\gamma=0.1$.

We realize from this expression that the time window can exist only if $\beta>2 \alpha$ and increases in size, as well as coupling strength $g_{\text {eff }}$ decreases.

In this time window, where no entanglement is found in subsystems $a_{1} \otimes a_{2}, c_{1} \otimes c_{2}$ and $r_{1} \otimes r_{2}$, the question of where the entanglement goes in this time window becomes relevant. In order to answer this question we must analyze the entanglement present between other bipartite subsystems of the overall system.

First we consider the subsystem $\left(a_{1}, c_{1}, r_{1}\right) \otimes$ $\left(a_{2}, c_{2}, r_{2}\right)$. Since this bipartite subsystem is in a pure state at all times, we are able to quantify the entanglement through the square root of the tangle [26]. This entanglement is given by:

$$
\sqrt{\tau_{12}(t)}=2 \alpha \beta
$$

which corresponds to the same amount of entanglement initially present in the atomic subsystem. This conservation of the global entanglement occurs due to the non-interacting character of the subsystem $\left(a_{1}, c_{1}, r_{1}\right) \otimes$ $\left(a_{2}, c_{2}, r_{2}\right)$ For interacting subsystems, for example, $a_{1(2)} \otimes c_{1(2)}$ and $c_{1(2)} \otimes r_{1(2)}$, concurrences are shown in Fig. 10, From the figure, we observe that in the time window where no entanglement is found in subsystems $a_{1} \otimes a_{2}$ and $r_{1} \otimes r_{2}$, the interacting subsystems show entanglement. Indeed, in such a time window, the entanglement seems to flow only through interacting subsystems. To confirm this, we need to consider different non-interacting subsystems such as, $a_{1} \otimes c_{2}, a_{1} \otimes r_{2}$ and $c_{1} \otimes r_{2}$. In these cases the concurrences are respectively

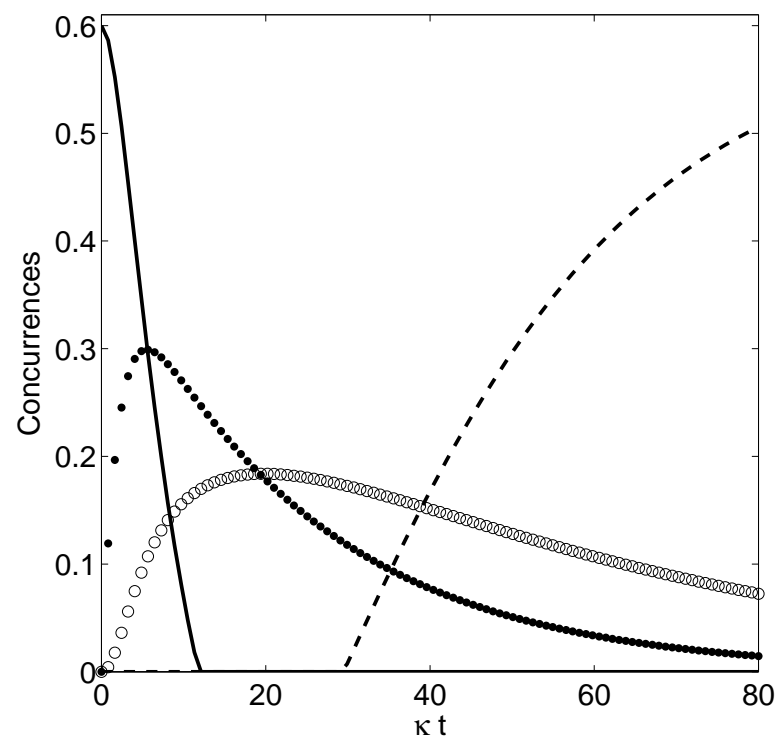

FIG. 10. Evolution of concurrence of the subsystems $a_{1} \otimes$ $c_{1}$ (dots), $c_{1} \otimes r_{1}$ (circles), $a_{1} \otimes a_{2}$ (solid line) and $r_{1} \otimes r_{2}$ (dashed line) for $\beta=3 \alpha$ and $\gamma=0.1$.

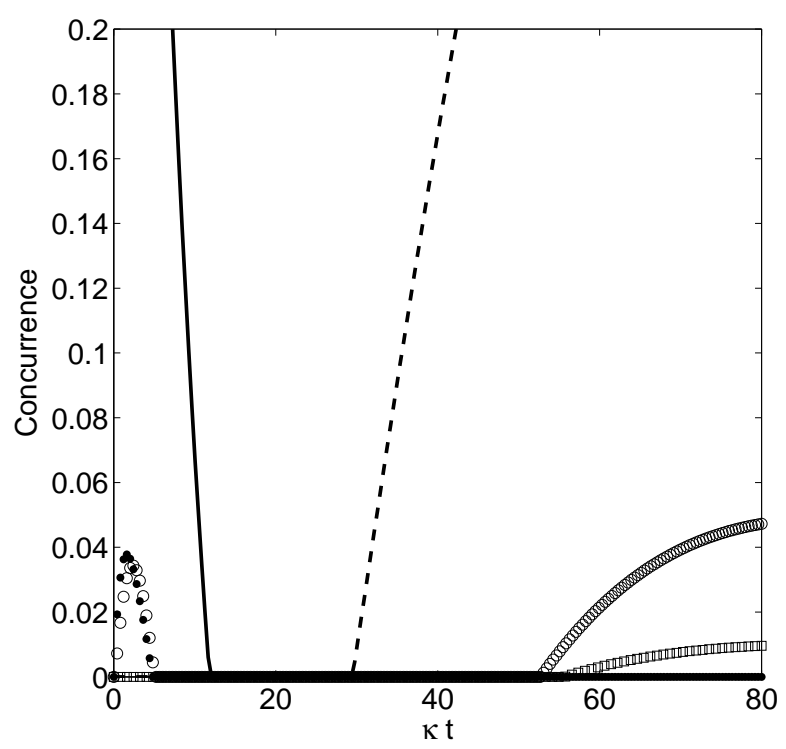

FIG. 11. Evolution of concurrence of the subsystems $a_{1} \otimes c_{2}$ (dots), $a_{1} \otimes r_{2}$ (circles), $c_{1} \otimes r_{2}$ (squares), $a_{1} \otimes a_{2}$ (solid line) and $r_{1} \otimes r_{2}$ (dashed line) for $\beta=3 \alpha$ and $\gamma=0.1$.

given by:

$$
\begin{aligned}
& \mathcal{C}_{a_{1} c_{2}}(t)=\max \left\{0,2\left[\alpha \beta E_{t}\left|G_{t}\right|-\sqrt{w\left(E_{t},\left|G_{t}\right|\right)}\right]\right\} \\
& \mathcal{C}_{a_{1} r_{2}}(t)=\max \left\{0,2\left[\alpha \beta E_{t} R_{t}-\sqrt{w\left(E_{t}, R_{t}\right)}\right]\right\} \\
& \mathcal{C}_{c_{1} r_{2}}(t)=\max \left\{0,2\left[\alpha \beta\left|G_{t}\right| R_{t}-\sqrt{w\left(\left|G_{t}\right|, R_{t}\right)}\right]\right\}
\end{aligned}
$$

where $w(x, y)=\alpha^{4} x y^{2}\left(1-x^{2}\right)\left(1-y^{2}\right)$. The temporal evolution of these concurrences is shown in Fig. [11. According to this figure, in the aforementioned time window, no contribution to entanglement comes from such 
non-interacting subsystems. Moreover, the time window where no entanglement is found in this subsystems is longer than the respective time window for the noninteracting subsystems $a_{1} \otimes a_{2}$ and $r_{1} \otimes r_{2}$. This confirms that in this finite time window the entanglement can flow only through interacting subsystems, such as $a_{1(2)} \otimes c_{1(2)}$ and $c_{1(2)} \otimes r_{1(2)}$, while the non-interacting subsystems have no entanglement.

\section{SUMMARY}

In summary, we have studied the dynamics of the entanglement transfer of two uncoupled systems each composed of a single atom inside a leaky cavity. We have developed a hybrid analytical approach to find the entanglement dynamics without tracing out reservoir modes, allowing us to study the entanglement behavior in dif- ferent subsystems. In particular, we have found that the entanglement initially located in two atoms is asymptotically mapped to the reservoirs degrees of freedom. Moreover, although reservoirs are connected to atoms through the cavities, we show that for a set of initial amplitudes and coupling constants, there are entangled and unentangled phases in the cavities. We have also shown that there is a time window where no entanglement is found in non-interacting subsystems such us atoms, cavities or reservoir. In this case, we observe that the entanglement flows only through interacting subsystems.

CEL acknowledge financial support from Fondecyt 11070244 and PBCT-CONICYT PSD54, GR from Juan de la Cierva Program, JCR from Fondecyt 1070157. CEL and JCR akcnowledge support from Financiamiento Basal para Centros Cientícos y Tecnológicos de Excelencia.
[1] D. Bouwmeester, A. Ekert, \& A. Zeilinger, The Physics of Quantum Information, Springer, Berlin, 2008.

[2] T. S. Cubitt, F. Verstraete, and J. I. Cirac, Phys. Rev. A 71, 052308 (2005).

[3] D. Cavalcanti, J.G. Oliveira, Jr., J. G. Peixoto de Faria, M. O. Terra Cunha and M. F. Santos, Phys. Rev. A 74, 042328 (2006).

[4] S. Chan, M.D. Reid, and Z. Ficek, arXiv: 0811.4466 (2008).

[5] M. Yonac, Ting Yu, J. H. Eberly, J. Phys. B 40, S45 (2007).

[6] C.E. López, G. Romero, F. Lastra, E. Solano and J. C. Retamal, Phys. Rev. Lett. 101, 080503 (2008).

[7] C.E. López, H. Christ, J.C. Retamal and E. Solano, Phys. Rev. A 75, 033818 (2007).

[8] C.E. López, F. Lastra, G. Romero and J. C. Retamal, Phys. Rev. A 75, 022107 (2007).

[9] M.O. Scully and M.S. Zubairy, Quantum Optics, (Cambridge University Press, Cambridge, 1997)

[10] C. Di Fidio, W. Vogel, M. Khanbekyan and D.-G. Welsch. Phys. Rev. A 77, 043822 (2008).

[11] J.I. Cirac, P. Zoller, H.J Kimble, and H. Mabuchi, Phys. Rev. Lett. 78, 3221, (1997).

[12] A. Serafini, S. Mancini, and S. Bose, Phys. Rev. Lett. 96, 010503 (2006).
[13] Chang-shui Yu, X. X. Yi, He-shan Song and D. Mei, Phys. Rev. A 75, 044301 (2007).

[14] Chang-shui Yu, X. X. Yi, He-shan Song and D. Mei, European Journal of Physics D 48, 411(2008)

[15] S. Zippilli, G. A. Olivares-Renteria, G. Morigi, C. Schuck, F. Rohde, J. Eschner, New J. Phys. 10, 103003 (2008).

[16] X. Wang, S. G. Schirmer, Phys. Rev. A 80, 042305 (2009).

[17] C. Di Fidio, W. Vogel, e-print arXiv:0908.1491 1 [quant$\mathrm{ph}$ ] (2009).

[18] C. Russo, et. al, Appl. Phys 95, 205 (2009).

[19] W. K. Wootters, Phys. Rev. Lett. 80, 2245 (1998).

[20] A. Peres, Phys. Rev. Lett. 77, 1413 , (1996).

[21] M. Horodecki, P. Horodecki, R. Horodecki, Phys. Lett. A 223, 1 (1996).

[22] M. F. Santos, P. Milman, L. Davidovich, and N. Zagury, Phys. Rev. A. 73, 040305(R), 2006.

[23] K. Życzkowski, P. Horodecki, M. Horodecki, and R. Horodecki. Phys. Rev. A 65, 012101 (2001).

[24] L. Diósi, Lect. Notes Phys. 622, 157-163 (2003).

[25] Ting Yu and J. H. Eberly, Phys. Rev. Lett. 93, 140404 (2004); ibid. 97, 140403 (2006).

[26] P. Rungta, V. Buzek, C.M. Caves, M. Hillery, and G.J. Milburn, Phys. Rev. A 64, 042315 (2001). 